Baltic Astronomy, vol. 9, 569-572, 2000

\title{
CATALOGUE OF GALACTIC STAR-FORMING REGIONS. OBSERVATIONAL DATA
}

\section{Avedisova}

Institute of Astronomy, Russian Academy of Sciences, Pyatnitskaja str. 48, Moscow 109017, Russia

Received November 10, 2000.

Abstract. The presented catalogue of "Star-forming regions in the Galaxy. Observational data" contains positions, sizes, fluxes and radial velocities of young objects in 3290 regions of star formation. The photometric and kinematic data on sources are accompanied by the information on diffuse and reflecting nebulae, dark and molecular clouds and different objects connected with young stars.

Key words: catalogs - Galaxy: star-forming regions, nebulae, dark clouds - stars: pre-main-sequence

\section{INTRODUCTION}

The star-forming region (SFR) is a site where young stellar objects (YSOs) are observed. They can include: (1) diffuse nebulae, $\mathrm{H}$ II regions and ultracompact H II regions, (2) pre-main-sequence stars with large infrared excesses, (3) infrared sources with negative (or small positive) spectral indexes, (4) non-stellar molecular maser sources, (5) reflection nebulae and Herbig-Haro objects in dark-cloud complexes, (6) molecular clouds, cores, clumps, hot spots and globules.

The catalogue contains: $\sim 3300$ SFRs, which include $\sim 3600$ IRAS sources, $\sim 530 \mathrm{OH}$ maser sources, $\sim 700 \mathrm{H}_{2} \mathrm{O}$ maser sources, $\sim 700 \mathrm{CH}_{3} \mathrm{OH}$ maser sources and 5098 bibliographical references.

CSFR gives the following data:

- photometric and kinematic characteristics of SFRs in radio and infrared ranges, 
- fluxes and radial velocities of non-stellar maser sources,

- limited information on optical and dark nebulae and molecular clouds, associated with the SFRs.

The data on SFRs in the large nearest cloud complexes were separated into the Extension because these complexes cover many degrees in the sky. Extension is a set of 17 table-files with the format which is similar to the Catalogue one. The Extension includes 13 nearest large complexes with distances generally within $500-700 \mathrm{pc}$ from the Sun:

1. Auriga cloud complex

8. Ophiuchus cloud complex

2. Cepheus cloud complex

9. Orion cloud complex

3. Chamaeleon cloud complex

4. Coalsack cloud

10. Perseus cloud complex

5. Corona Australis cloud

11. Serpens cloud

6. Gum nebula

12. Taurus cloud complex

7. Lupus cloud complex

13. Vela cloud complex

\section{TWO TYPES OF STAR-FORMING REGIONS}

It is well known that star-forming regions may be dominated either by massive stars, or by low-and intermediate-mass stars.

The birth of massive stars is accompanied by strong stellar wind with the velocities up to a few thousand $\mathrm{km} / \mathrm{s}$. The wind creates cavities with diameter of about $1000 \mathrm{AU}$ which are observed as ultracompact (UC) H II regions of various morphological classes indicated in the catalogue.

The massive young objects are often associated with the maser emission sources such as $\mathrm{OH}, \mathrm{H}_{2} \mathrm{O}$ and $\mathrm{CH}_{3} \mathrm{OH}$ masers. Due to their high luminosity they are the best tracers of the UC H II regions

The formation of low and intermediate mass stars is generally observed in dense cores of dark clouds and in globules. These young objects are usually classified into four classes according to the shapes of their spectra in the near- to mid-infrared and following an evolutionary sequence of sources:

- Class 0 refers to the most deeply embedded sources, undetected in optical and in the near/mid-IR. Such an object corresponds to the youngest protostar stage observed. 
- Class I refers to embedded "protostellar" objects, invisible in the optical with steeply rising spectra longward of $2 \mu \mathrm{m}$.

- Class II corresponds to optically visible stars with flat or decreasing spectra longward of $2 \mu \mathrm{m}$, with strong $\mathrm{H} \alpha$ line emission. They are considered as PMS stars (classical T Tau stars (CTTSs) and Ae/Be stars) with a strong infrared excess from a circumstellar disk.

- Class III refers to stars exhibiting a weak infrared excess over a reddened stellar blackbody. They are considered as diskless PMS stars on their way to the main sequence (weak-line T Tau stars (WTTSs) or/and strong X-ray emitters with relatively weak $\mathrm{H} \alpha$ line). The catalogue includes, as a rule, the objects of $0, \mathrm{I}$ and partly II classes.

\section{FORMAL CLASSIFICATION OF SFRs}

A star-forming region can include the following physical objects:

- own forming star;

- H II regions generated around high mass young stars;

- accretion discs and cocoons formed around young stars and embedded in the parent cloud;

- different phenomena accompanying the star formation process such as masers, Herbig-Haro objects, jets, bipolar nebulae, molecular outflows;

- dark molecular clouds or cloud cores as parental matter from which stars form.

Each of these sources is responsible for the certain type(s) of information which is presented in the catalogue. We have five such types:

- the data on the dark nebula or globula associated with the SFR are marked as type $D$;

- the data on the H II regions observed in radio continuum and radio recombination lines are marked as type $R$;

- the optical data on diffuse and reflection nebula, Herbig-Haro objects and visible stars are marked as type $\mathrm{O}$;

- the data on the accretion discs, cocoons or/and dense clouds connected with YSO observed in the infrared are marked as type IR; 
- the data on the molecular clouds, molecular outflows and masers observed in various molecular lines are marked as type $M$.

Each SFR may be classified according to a set of data types which is called as "class of SFR". So, the class 'RIM' means that the data of the SFR include ' $R$ ', 'IR' and ' $M$ ' types; the class 'OIM' means that the data include ' $O$ ', 'IR' and ' $M$ ' types, etc.

The frequency of different classes of a sample of distant SFRs are determined. Three classes of objects dominate in this sample: IM, RIM and ORIM: IM counts 971 regions, RIM - 622 regions and ORIM - 305 regions. The analysis of distributions of these classes shows that RIM and ORIM classes are connected with the regions of formation of massive stars. IM class is composed of two families of the SFRs. The longitude distribution of RIM class objects and a sample from it associated with $\mathrm{H}_{2} \mathrm{O}$ masers are in good correlation following the $\chi^{2}$ criterion. The ratio of the total number of RIM objects to the number of RIM objects associated with $\mathrm{H}_{2} \mathrm{O}$ masers is 4.7. The same holds for the ORIM class objects. It is amazing that the ratio of the total number of ORIM objects to the number of ORIM objects associated with $\mathrm{H}_{2} \mathrm{O}$ masers is 4.9 , very close to the above ratio for RIM objects. The coincidence of two independent samples suggests that these ratios reflect the intrinsic statistics of SFRs with $\mathrm{H}_{2} \mathrm{O}$ masers rather than selection effects and indicates likely to the real ratio of SFRs with and without masers. If so, we may assume that this ratio corresponds to the ratio of the overall life time of RIM and ORIM star-forming regions to the life time of $\mathrm{H}_{2} \mathrm{O}$ masers stage, which thus turns out to be $20 \%$ of the life time of compact H II region. 